\title{
ON THE FINITENESS AND UNIQUENESS OF CERTAIN 2-TAME $N$-GROUPS
}

\author{
by S. D. SCOTT
}

(Received 5th July 1993)

\begin{abstract}
Unlike ring modules certain faithful $N$-groups are unique. The main theorem is that if $N$ is a 2-tame ring-free near-ring where $N / J(N)$ has DCCR, then all faithful 2-tame $N$-groups are finite and $N$-isomorphic. The finiteness of such an $N$-group follows easily from the fact it has a composition series. It is then shown that the length of a composition series depends only on $N$. This fact is used at key points in the proof. The situations where the $N$-group has or has not a minimal submodule require different analysis. The first case makes use of other interesting results and the second makes strong use of the inductive assumption.
\end{abstract}

1991 Mathematics subject classification: 16 Y30.

Throughout this paper all near-rings are left distributive, zero-symmetric and have an identity. Also all $N$-groups will be unitary. In this and other regards we shall be making use of conventions, notation and definitions taken from [5]. The purpose of this paper is to prove the following theorem.

Theorem 1. Let $N$ be a 2-tame ring-free near-ring. If $N / J(N)$ has $D C C R$, then all faithful 2-tame $N$-groups are finite and $N$-isomorphic.

Although the definition of all terms used in the statement of this theorem can be found in [5], it remains desirable, for the purposes of completeness, to briefly explain their meaning.

A near-ring $N$ is said to have $D C C R$ if it satisfies the descending chain condition on right ideals. In the above theorem it is only required that $N / J(N)$ satisfies this condition but strong consequences follow. The meaning associated with the statement ' $N$ is ringfree' is that no non-zero homomorphic image of $N$ is a ring.

A tame $N$-group $V$ is one in which all $N$-subgroups are submodules. This is equivalent (see 2.1 of [5]) to requiring that, for any given $v$ and $w$ in $V$ and $\alpha$ in $N$, we can find $\beta$ in $N$ such that $(v+w) \alpha-v \alpha=w \beta$. This characterization of a tame $N$-group leads to the definition of $n$-tame, where $n$ is any cardinal (see 9.169 of [3]). For example, a 2-tame $N$-group $V$ is one where, for given $v$ in $V$ and $\alpha$ in $N$, we may find $\beta$ in $N$, such that $\left(v+w_{i}\right) \alpha-v \alpha=w_{i} \beta$ for any pair $\left(w_{1}, w_{2}\right)$ of elements of $V$. Furthermore, a near-ring $N$ with a faithful tame (2-tame) $N$-group is called tame (2-tame).

On p. 242 of [4], we define the centre $Z(V)$ of an $N$-group $V$. Also the useful concept of a central sum is defined in p. 243. The $N$-group $V$ is said to be a central sum of the 
submodules $V_{i}, i=1,2$, if $V=V_{1}+V_{2}$ and $\left(v_{1}+v_{2}\right) \alpha=v_{1} \alpha+v_{2} \alpha$, for all $v_{i}, i=1,2$, in $V_{i}$ and $\alpha$ in $N$. If this is the case, then $V_{1} \cap V_{2} \leqq Z(V)$.

The proof of the above theorem will be accomplished in the sequence of results that follow. The easiest part of the proof is to establish that the $N$-groups involved are finite.

According to 5.32 of [3], the semi-simple near-ring $N / J(N)$ is a direct sum $R_{1} \oplus R_{2} \oplus \cdots \oplus R_{k}(k \geqq 1$ an integer) of minimal right ideals. If $U$ is a minimal $N$-group, then it is a minimal $N / J(N)$-group. It follows that $U R_{i} \neq\{0\}$ for some $i$ in $\{1, \ldots k\}$ and thus $u R_{i}=U$ for some $u$ in $U$. From this it is easily verified that the map taking $\rho$ in $R_{i}$ to $u \rho$ in $u R_{i}$ is an $N$-isomorphism of $R_{i}$ onto $U$. Thus the number of $N$-isomorphism types of minimal $N$-groups cannot exceed $k$. Let this finite number be denoted by $m(N)$.

Proposition 2. Under the assumptions of Theorem 1 the number $m(N)$ of distinct $N$-isomorphism types of minimal $N$-groups is finite

The next lemma is one of the main steps in establishing the finiteness of a faithful 2-tame $N$-group.

Lemma 3. Let $N$ be as in the statement of Theorem 1. If $V$ is a faithful 2-tame $N$-group, then $V$ has a composition series of length $\leqq m(N)$.

Proof. Suppose $V$ is non-zero and does not have a composition series. A series of submodules.

$$
V_{0}=\{0\}<V_{1}<\cdots<V_{r}=V
$$

of $V$, with $r \geqq 1$ an integer, will have a proper refinement and the series obtained in turn has a proper refinement, etc. It follows readily that, if $V$ does not have a composition series, or there exists a composition series of length $>m(N)$, then the series (a) can be chosen so that $r>m(N)$.

Now take $v_{i}$ in $V_{i}$ but not in $V_{i-1}$ for $i=1, \ldots, r$. Using Zorn's lemma we can find a submodule $X_{i}$ of $V_{i}$ containing $V_{i-1}$ and maximal for excluding $v_{i}$. Let $Y_{i}=v_{i} N+X_{i}$. It follows that for each $i=1, \ldots, r, V_{i-1} \leqq X_{i}<Y_{i} \leqq V_{i}$ and $Y_{i} / X_{i}$ is a minimal factor of $V$. Since $r>m(N)$, there exists $j$ and $k, j<k$, in $\{1, \ldots r\}$ such that $Y_{k} / X_{k}$ is $N$-isomorphic (by $\sigma$ say) to $Y_{j} / X_{j}$. It is also clear that

$$
X_{j}<Y_{j} \leqq V_{k-1} \leqq X_{k}<Y_{k}
$$

Let $\kappa$ be the natural $N$-homomorphism of $Y_{k} / X_{j}$ onto

$$
\left(Y_{k} / X_{j}\right) /\left(X_{k} / X_{j}\right)
$$

Since this $N$-group is $N$-isomorphic (by $\delta$ say) to $Y_{k} / X_{k}, \kappa \delta \sigma$ is an $N$-endomorphism of $Y_{k} / X_{j}$ onto $Y_{j} / X_{j}$. By 7.3 of [5], $1-\kappa \delta \sigma$ is an $N$-endomorphism of $Y_{k} / X_{j}$ and, by 1.4 of 
[4], $Y_{k} / X_{j}$ is a central sum of the submodules $\left(Y_{k} / X_{j}\right) \kappa \delta \sigma$ and $\left(Y_{k} / X_{j}\right)(1-\kappa \delta \sigma)$. Now $\left(Y_{k} / X_{j}\right) \kappa \delta \sigma=Y_{j} / X_{j}$. Also $\operatorname{ker} \kappa \delta \sigma=k e r \kappa=X_{k} / X_{j}$ and

$$
\left(X_{k} / X_{j}\right)(1-\kappa \delta \sigma)=\left(X_{k} / X_{j}\right)
$$

Thus

$$
\left(Y_{k} / X_{j}\right) \kappa \delta \sigma \cap\left(Y_{k} / X_{j}\right)(1-\kappa \delta \sigma) \geqq Y_{j} / X_{j}
$$

It follows, by 1.3 of [4], that $Y_{j} / X_{j}$ is a central submodule of $Y_{k} / X_{j}$. Thus $N /\left(0: Y_{j} / X_{j}\right)(\neq\{0\})$ is a ring. This contradiction implies $V$ has a composition series of length $\leqq m(N)$. The lemma is entirely proved.

Our next requirement is a result that depends on 8.4 of [5] (see also 1.5 of [4]). A relatively straightforward proof, based on 8.4 of [5], can be found in [6] (see also 4.61 of [3]).

Theorem 4. Suppose the non-ring $N$ is 2-tame and primitive on $V$. If $N$ has DCCR, then $V$ is finite.

The proof that the $N$-groups of Theorem 1 are finite now follows readily.

Lemma 5. Let $N$ be as in the statement of Theorem 1. If $V$ is a faithful 2-tame $N$-group, then $V$ and $N$ are finite.

Proof. If $V \neq\{0\}$, then by Lemma 3 , there exists an integer $r \geqq 1$ and composition series

$$
V_{0}=\{0\}<V_{1}<V_{2}<\cdots<V_{r}=V
$$

of submodules of $V$. Since $V_{i} / V_{i-1}, i=1, \ldots, r$, are minimal $N$-groups the primitive non-rings $N /\left(0: V_{i} / V_{i-1}\right)$ are homomorphic images of $N / J(N)$. Therefore they have DCCR. Clearly they are 2-tame on $V_{i} / V_{i-1}$ for $i=1, \ldots, r$. Thus, by 4 , each $V_{i} / V_{i-1}$, $i=1, \ldots, r$, is finite. Thus $V$ is finite. Since $N$ can be regarded as a subnear-ring of $M_{0}(V), N$ must be finite also. The lemma is completely proved.

We now come to a much more difficult aspect, that of proving that all $N$-groups, as in the statement of Theorem 1 , are in fact $N$-isomorphic.

At this stage it is possible to sharpen Lemma 3. The more precise information given in the next lemma will be required later.

Lemma 6. Let $N$ be as in the statement of Theorem 1. If $V$ is a faithful 2-tame $N$-group, then the length of a composition series of $V$ is $m(N)$.

Proof. Suppose 


$$
V_{0}=\{0\}<V_{1}<V_{2}<\cdots<V_{r}=V
$$

is a composition series of $V$ ( $r \geqq 1$ being an integer). By Lemma 3, we have $r \leqq m(N)$. If it is shown that for any minimal $N$-group $U$ there exists a minimal factor $Y / X$ of $V, N$ isomorphic to $U$, then, since $Y / X$ is $N$-isomorphic to some $V_{i} / V_{i-1}(i$ in $\{1, \ldots, r\}$ ), it will follow that $r \geqq m(N)$ and $r=m(N)$.

As in the explanation proceeding Proportion 2 we have that $U$ is $N$-isomorphic to a minimal right ideal of $N / J(N)$. This right ideal is clearly of the form $R / J(N)$, where $R>J(N)$ is a right ideal of $N$. By Lemma $5, N$ is finite and out of all minimal factors of $N, N$-isomorphic to the minimal factor $R / J(N)$, we may choose one $H / K$ where $H$ is minimal. Now if $K_{1}<H$ is a right ideal of $N$ such that $K_{1} \leq K$, then $K_{1}+K=H$ and $H / K$ is $N$-isomorphic to the minimal factor $K_{1} / K_{1} \cap K$. Thus $K_{1} \leqq K$. Since $V$ is faithful there exists $v$ in $V$ such that $(0: v) \cap H<H$. Consequently $(0: v) \cap H \leqq K$.

Let $\delta$ be the obvious $N$-homomorphism of $H$ into $v N$. Let $\kappa$ be the natural $N$ homomorphism of $v H$ onto $v H / v K$. Since ker $\delta \kappa$ consists of all $\rho$ in $H$ such that $v \rho$ is in $v K$, it contains $K$. However, $k e r \delta \kappa$ is an $N$-subgroup of $H$ and therefore, by 4.2 of [5], $k e r \delta \kappa=K$ or $k e r \delta \kappa=H$. Suppose $k e r \delta \kappa=H$. In this case $v H=v K$ and for each $\alpha$ in $H$ there exists $\beta$ in $K$ such that $v \alpha=v \beta$. This implies $H \leqq K+(0: v)$, and therefore $H=K+(0: v) \cap H$. However, from above $(0: v) \cap H \leqq K$ and we have the contradiction that $H=K$. Thus $k e r \delta \kappa=K$ and $v H / v K$ is $N$-isomorphic to $H / K$ which is $N$-isomorphic to $U$. Thus $V$ has a minimal factor $N$-isomorphic to $U$ and, according to the explanation given at the beginning of the proof $r=m(N)$. The lemma is completely proved.

More detailed information on the structural properties of tame $N$-groups of a ring-free near-ring $N$ is now developed.

Theorem 7. Suppose $N$ is a ring-free near-ring. If $V$ is a tame $N$-group with a composition series, then there exists $v$ in $V$ such that $v N=V$.

Proof. Clearly $V$ satisfies both ascending and descending chain conditions on submodules. It follows that if $V$ is not cyclic (i.e. monogenic), then there exists a minimal non-cyclic submodule $U$ of $V$. Obviously $U \neq\{0\}$. Suppose the sum of any two proper submodules $H_{i}, i=1,2$, of $U$ is such that $H_{1}+H_{2}<U$. If $H$ is a maximal submodule of $\mathrm{U}$, then $\mathrm{H}+\mathrm{H}_{2}=\mathrm{H}$ and $\mathrm{H}_{2} \leqq H$. In this case $H$ must be the unique maximal submodule of $U$. Take $v$ in $U \backslash H$. Clearly $v N$ is not contained in $H$. This implies $v N=U$. Thus there exist proper submodules $H_{i}, i=1,2$, of $U$, such that $H_{1}+H_{2}=U$. Out of all such pairs of submodules choose one $K_{i}, i=1,2$, with $K_{1} \cap K_{2}$ minimal. Now $K_{1}+K_{2}=U$ and there exists $v_{i}, i=1,2$, in $K_{i}$ such that $v_{i} N=K_{i}$. Since the $v_{i}, i=1,2$, are contained in $U$, it follows that

$$
\left(v_{1}+v_{2}\right) N+v_{i} N \leqq U .
$$

However, $\left(v_{1}+v_{2}\right) N+v_{1} N$ contains $v_{2} N$. Similarly $\left(v_{1}+v_{2}\right) N+v_{2} N$ contains $v_{1} N$. Since $K_{1}+K_{2}=U$, it follows that 


$$
\left(v_{1}+v_{2}\right) N+v_{i} N=U
$$

for $i=1$ and 2. Since $N /(0: V)$ is ring-free, it follows by 3.24 of [2] that the lattice of submodules of $V$ is distributive. Thus

$$
\left(v_{1}+v_{2}\right) N+\left(v_{1}+v_{2}\right) N \cap v_{1} N+\left(v_{1}+v_{2}\right) N \cap v_{2} N+v_{1} N \cap v_{2} N=U
$$

Clearly

$$
\left(v_{1}+v_{2}\right) N \cap v_{i} N \leqq\left(v_{1}+v_{2}\right) N
$$

for $i=1,2$, and it therefore follows that

$$
\left(v_{1}+v_{2}\right) N+v_{1} N \cap v_{2} N=U
$$

However, if

$$
\left(v_{1}+v_{2}\right) N \cap v_{1} N \cap v_{2} N<v_{1} N \cap v_{2} N
$$

then we have a contradiction to the choice of $K_{i}\left(=v_{i} N\right), i=1,2$. Thus

$$
\left(v_{1}+v_{2}\right) N \geqq v_{1} N \cap v_{2} N
$$

and, by (b), $U=\left(v_{1}+v_{2}\right) N$. This contradiction to the nature of $U$ establishes that the $N$-group $V$ is cyclic. Theorem 7 is entirely proved.

Suppose $N$ is as in the statement of Theorem 1. In proving that all faithful 2-tame $N$-groups are $N$-isomorphic there are two cases to be distinguished. These are the situations where $N$ has more than one minimal ideal and where $N$ has a unique minimal ideal. We deal with the second case first. This property of $N$ can be recovered from a faithful 2-tame $N$-group.

Lemma 8. Let $N$ be as in the statement of Theorem 1. A faithful 2-tame $N$-group has a unique minimal submodule if, and only if, $N$ has a unique minimal ideal.

Proof. Let $V$ be a faithful 2-tame $N$-group. Suppose $V$ has a unique minimal submodule $U$. By Lemma $5, N$ has $D C C$ on $N$-subgroups and therefore has a minimal ideal $T$ say. From 3.54 of [3], $T$ can be expressed as a direct sum $R_{1} \oplus R_{2} \oplus \cdots \oplus R_{k}$ ( $k \geqq 1$ an integer) of minimal right ideals. Also, by 4.2 of [5], these minimal right ideals are minimal right $N$-subgroups of $N$. If $x$ is in $V$, then $x T=\sum_{i=1}^{k} x R_{i}$. Furthermore, each $x R_{i}=\{0\}$ or is $N$-isomorphic to $R_{i}$. Thus, if $x R_{i} \neq\{0\}$, then it is a minimal $N$-subgroup of $V$ and coincides with $U$. It follows that $x T=\{0\}$ or $x T=U$.

Now suppose $T_{1}$ and $T_{2}$ are two distinct minimal ideals of $N$. By Theorem 7, $V=v N$ for some $v$ in $V$. Since $V T_{i} \neq\{0\}$ for $i=1,2$, we have, from above, $v T_{1}=v T_{2}=U$. However, since the sum $T_{1}+T_{2}$ is direct, $U$ is a central sum of $v T_{i}, i=1,2$. By 1.3 of [4], 
this implies $U$ is a ring module and $N /(0: U)$ is a ring. Thus $N$ has a unique minimal ideal.

Suppose on the other hand $N$ has a unique minimal ideal. Clearly $V \neq\{0\}$ and, by 5 , $V$ has minimal submodules. To obtain a contradiction assume, that $U_{i}, i=1,2$, are distinct minimal submodules of $V$. If $\left(U_{1}: V\right)=\{0\}$, then $N$ is faithful on the 2-tame $N$-group $V / U_{1}$. By Lemma 6 this implies that $V / U_{1}$ has a composition series of length $m(N)$. However, since $N$ is faithful on $V, V$ also has a composition series of length $m(N)$. This contradiction means $\left(U_{1}: V\right) \neq\{0\}$. Similarly $\left(U_{2}: V\right) \neq\{0\}$. Clearly

$$
\left(U_{1}: V\right) \cap\left(U_{2}: V\right) \leqq(0: V)=\{0\} .
$$

By Lemma $5, N$ has minimal ideals $T_{i}, i=1,2$, contained in $\left(U_{i}: V\right)$. However, since $T_{1} \cap T_{2}=\{0\}$ the $T_{i}$ are distinct. This contradiction implies $V$ has a unique minimal submodule. The lemma is completely proved.

Let $N$ be as in the statement of Theorem 1. We come now to the main step in showing that, when $N$ has a unique minimal ideal, all faithful 2-tame $N$-groups are $N$ isomorphic. In order to state this lemma it is convenient at this stage to introduce the centralizer of a right ideal of a ring-free near-ring.

If $N$ is a ring-free near-ring and $R$ a right ideal of $N$, then, by Zorn's lemma, there exists a right ideal $H$ of $N$ maximal for the property that $H \cap R=\{0\}$. Furthermore, $H$ is unique since if $H_{1}$ is a right ideal of $N$ such that $H_{1} \cap R=\{0\}$, then, by 3.24 of [2], $R \cap\left(H_{1}+H\right)=\{0\}$. Thus $H_{1}+H=H$ and $H_{1} \leqq H$. We shall denote the right ideal $H$ of $N$ by $C_{N}(R)$. This will be called the centralizer of $R$ in $N$. This name is given to $H$ since, as is easily verified, it is the unique right ideal of $N$ maximal for the property that $(\rho+h) \alpha=\rho \alpha+h \alpha$ for all $\rho$ in $R, h$ in $H$ and $\alpha$ in $N$.

Lemma 9. Let $N$ be as in the statement of Theorem 1. Suppose $N$ has a unique minimal ideal $T$ and $R \leqq T$ is a minimal right ideal of $N$. If $V$ is a faithful 2-tame $N$-group, then $V$ is $N$-isomorphic to $N / C_{N}(R)$.

Proof. Since $V$ is a faithful $N$-group there exists $v$ in $V$ such that $v R \neq\{0\}$. We first show that $v N=V$. Clearly $v N T \supseteq v R \neq\{0\}$. Thus $T \cap(0: v N)=\{0\}$. However, by Lemma $5, N$ is finite and if $(0: v N) \neq\{0\}$, then $(0: v N)$ contains a minimal ideal. This contradiction implies $(0: v N)=\{0\}$. Thus $v N$ is a faithful 2-tame $N$-group and $\{0\} \leqq v N \leqq V$. Since by Lemma $6, v N$ and $V$ both have composition series of length $m(N)$, it follows that $v N=V$.

Now let $\delta$ be the obvious $N$-homomorphism of $N$ onto $v N$. Since $\operatorname{ker} \delta=(0: v)$ the lemma will follow if it is shown that $(0: v)=C_{N}(R)$. By 4.2 of [5], $R$ is a minimal $N$-group, since $v R \neq\{0\}$ the map taking $\rho$ in $R$ to $v \rho$ is an $N$-isomorphism of $R$ onto $v R$. Thus $v R$ is a minimal $N$-group. However, the sum $R+C_{N}(R)$ is direct and, $v R+v C_{N}(R)$ is a central sum of $v R$ and $v C_{N}(R)$ (see $\S .1$ of [4]). If $v C_{N}(R) \neq\{0\}$, then by Lemma 5 , $v C_{N}(R)$ contains a minimal $N$-subgroup of $V$. However, by Lemma 8 , this coincides with $v R$. In this case it follows, by 1.3 of [4], that $v R$ is central in $v R+v C_{N}(R)\left(=v C_{N}(R)\right)$. 
This yields the contradiction that $N /(0: v R)(\neq\{0\})$ is a ring. Thus $v C_{N}(R)=\{0\}$ and $C_{N}(R) \leqq(0: v)$. If $C_{N}(R)<(0: v)$, then, by the maximality of $C_{N}(R),(0: v) \geqq R$. This contradiction to the fact that $v R \neq\{0\}$ implies $(0: v)=C_{N}(R)$. The lemma is completely proved.

As will be seen later the above result in fact deals with the situation where $N$ has a faithful 2-tame $N$-group $V$ with a unique minimal $N$-subgroup. Results that follow are directed toward providing insight as to what happens when $V$ has more than one minimal $N$-subgroup.

Lemma 10. Let $N$ be a ring-free near-ring and $V$ a tame $N$-group. If $W_{i}, i=1,2$, are minimal $N$-isomorphic $N$-subgroups of $V$, then $W_{1}=W_{2}$.

Proof. Suppose $W_{1} \neq W_{2}$. In this case the sum $W_{1}+W_{2}$ is direct. Suppose $\delta$ is an $N$-isomorphism of $W_{1}$ onto $W_{2}$. Let $\Delta$ be the subset of $W_{1} \oplus W_{2}$ consisting of all $w+w \delta$ where $w$ is in $W_{1}$. Clearly $\Delta \neq\{0\}$. Furthermore the difference of two elements of $\Delta$ is in $\Delta$ and, if $\alpha$ is in $N$ and $w_{1}$ in $W_{1}$, then $\left(w_{1}+w_{1} \delta\right) \alpha=w_{1} \alpha+w_{1} \alpha \delta$ is again in $\Delta$. Thus $\Delta$ is an $N$-subgroup of $V$ and consequently a submodule. If for $x_{1}$ in $W_{1}, x_{1}+x_{1} \delta$ is in $W_{1}$, then $x_{1} \delta=0$ and $x_{1}+x_{1} \delta=0$. Thus $\Delta \cap W_{1}=\{0\}$ and similarly $\Delta \cap W_{2}=\{0\}$. It follows, from Section 1 of [4], that $\Delta$ is a central submodule of $W_{1} \oplus W_{2}$. Thus if $W_{1} \neq W_{2}$, $N /(0: \Delta)(\neq\{0\})$ is a ring. This contradiction implies $W_{1}=W_{2}$ and the lemma follows.

Let $N$ be as in the statement of Theorem 1 and $V$ a faithful 2-tame $N$-group. The requirement that $V$ has a minimal $N$-subgroup of given $N$-isomorphism type is, in fact, equivalent to a condition on $N$.

Lemma 11. Let $N$ be as in the statement of Theorem 1, $V$ a faithful 2-tame $N$-group and $U$ a minimal $N$-group. We have that $V$ has a minimal $N$-subgroup $N$-isomorphic to $U$ if and only if, $N$ contains a minimal right ideal $N$-isomorphic to $U$.

Proof. Suppose $X$ is a minimal $N$-subgroup of $V, N$-isomorphic to $U$. If $(X: V)=\{0\}$, then $V / X$ is a faithful 2-tame $N$-group. By Lemma $6, V / X$ has a composition series of length $m(N)$. However, by Lemma 6 , we have the contradiction that $V$ also has a composition series of length $m(N)$. We conclude that $(X: V) \neq\{0\}$. Now, from Lemma 5 , there exists a minimal right ideal $R \leqq(X: V)$. Since $V$ is faithful we may find $v$ in $V$ such that $v R \neq\{0\}$. Now $v R \leqq X$, and thus $v R=X$. The map taking $\rho$ in $R$ to $v \rho$ in $X$ is an $N$-isomorphism of $R$ onto $X$. Thus $R$ is a minimal right ideal $N$-isomorphic to $X$ and therefore to $U$.

Suppose on the other hand $R$ is a minimal right ideal of $N, N$-isomorphic to $U$. Since $V$ is faithful we can find $v$ in $V$ such that $v R \neq\{0\}$. The map that takes $\rho$ in $R$ to $v \rho$ in $v R$ is an $N$-isomorphism of $R$ onto $v R$. Thus $U$ is $N$-isomorphic to the minimal $N$ subgroup $v R$ of $V$. The proof of Lemma 11 is complete.

To proceed further we need some straightforward results on complete reducibility. A submodule $U$ of an $N$-group $V$ is said to be completely reducible in $V$ (c.f. 15.1 of 
[1]) if, for every submodule $U_{1}$ of $V$ contained in $U$, there can be found a submodule $U_{2}$ of $V$ such that $U_{1} \oplus U_{2}=U$.

It is an elementary fact that:

Proposition 12. If a submodule $U$ of an $N$-group $V$ is completely reducible in $V$, then any submodule $H \leqq U$ of $V$ is completely reducible in $V$.

The techniques used to prove 15.3 of [1] are available to show that:

Proposition 13. A non-zero submodule of an $N$-group $V$ is completely reducible in $V$ if, and only if, it is a sum of minimal submodules of $V$.

The socle, $\operatorname{soc} N$ of a near-ring $N$ is defined to be a sum of all minimal right ideals of $N$ when such right ideals exist. Otherwise $\operatorname{soc} N$ is taken to be $\{0\}$. Proposition 12 and Proposition 13 have been stated in order to present the following corollary:

Corollary 14. Let $N$ be a near-ring. A non-zero right ideal of $N$ contained in $\operatorname{soc} N$ is a sum of minimal right ideals.

Let $N$ be a near-ring and $U$ a minimal $N$-group. It will be of use to have notation for specifying certain right ideals of $N$ contained in soc $N$. In this regard $S(U)$ is taken to be the sum $\sum R_{i}$ over all minimal right ideals $R_{i}$ of $N, N$-isomorphic to $U$ provided such right ideals exist. If there are no such right ideals of $N$, then $S(U)$ is taken to be $\{0\}$.

Lemma 15. Let $N$ be as in the statement of Theorem 1 and $V$ a faithful 2-tame $N$-group. If $U$ is a minimal $N$-subgroup of $V$, then $S(U)=(U: V)$.

Proof. It will first be shown that $S(U) \leqq(U: V)$. If $S(U)=\{0\}$, then this inclusion holds. We may therefore suppose (see Lemma 11) that there exists minimal right ideals of $N, N$-isomorphic to $U$. Let $R$ be such a right ideal. It is easily seen that for each $v$ in $V, v R=\{0\}$ or $v R$ is $N$-isomorphic to the $N$-subgroup $U$ of $V$. This follows since if $v R \neq\{0\}$, then the map taking $\rho$ in $R$ to $v \rho$ in $v R$ is an $N$-isomorphism of $R$ onto $v R$. However, by Lemma $10, v R=U$ when $v R \neq\{0\}$. Thus for all $v$ in $V, v R \leqq U$ and $R \leqq(U: V)$. Since this is true for any such $R, S(U) \leqq(U: V)$.

It remains to show that $(U: V) \leqq S(U)$. Since

$$
V(U: V) J(N) \subseteq U \cdot J(N)=\{0\}
$$

it follows that $(U: V) J(N)=\{0\}$. However, by Lemma $5, N$ has $D C C R$. By 5.3 of [5], it follows that $(U: V) \leqq \operatorname{soc} N$. Clearly we may assume $(U: V) \neq\{0\}$. Now Corollary 14 implies that $(U: V)$ is a sum $\sum R_{i}, i \in I$, of minimal right ideals of $N$. However for each $R_{i}, i \in I$, there exists $v$ in $V$ such that $v R_{i} \neq\{0\}$. Since $R_{i} \leqq(U: V), v R_{i}=U$ and the map taking $\rho$ in $R_{\mathrm{i}}$ to $v \rho$ is an $N$-isomorphism of $R_{i}$ onto $U$. Thus $R_{i}$ is in $S(U)$ and $(U: V) \leqq S(U)$. The lemma is entirely proved. 
The completion of the proof of Theorem 1 will be given once information concerning the embedding of certain $N$-groups into direct sums has been obtained. This accounts for the next three lemmas. The result that follows covers well known properties of certain subdirect products (the proof is omitted). All the information covered will be required.

Lemma 16. Let $V$ be an $N$-group and $U_{j}, j=1,2$, submodules of $V$ such that $U_{1} \cap U_{2}=\{0\}$. Let $Y$ be the external direct sum of $V / U_{1}$ and $V / U_{2}$ and let $H_{1}=$ $\left(V / U_{1},\{0\}\right)$ and $H_{2}=\left(\{0\}, V / U_{2}\right)$. Furthermore let $X_{1}=\left(\left(U_{1}+U_{2}\right) / U_{1},\{0\}\right)$ and $X_{2}=$ $\left(\{0\},\left(U_{1}+U_{2}\right) / U_{2}\right)$ and $\delta$ be the map of $V$ into $Y$ given by $v \delta=\left(v+U_{1}, v+U_{2}\right)$ for all $v$ in $V$. We have that $H_{j}$ and $X_{j}, j=1,2$, are submodules of $Y$ and $Y$ is the internal direct sum $H_{1} \oplus H_{2}$. Furthermore, $\delta$ is an $N$-group embedding of $V$ into $Y$, such that $V \delta+H_{j}=Y$ and $V \delta \cap H_{j}=X_{j}$ for $j=1,2$.

Under conditions similar to those of Lemma 16 information is required as to how certain 2-tame $\mathrm{N}$-groups are embedded in $\mathrm{H}_{1} \oplus \mathrm{H}_{2}$. The next lemma facilitates the proof of a much more useful result i.e. Lemma 19.

Lemma 17. Let $N$ be a ring-free near-ring. Suppose the $N$-group $Y$ is a direct sum $H_{1} \oplus H_{2}$ of submodules $H_{j}, j=1,2$. If $T_{k}, k=1,2$, are 2-tame $N$-subgroups of $Y$ such that $T_{k}+H_{j}=Y$ and $T_{k} \cap H_{j}=\{0\}$ for $k=1,2$, and $j=1,2$, then $T_{1}=T_{2}$.

Proof. Let $\pi_{j}, j=1,2$, be the projection of $Y$ onto $H_{j}$. Let $\pi_{k j}$ be the restriction of $\pi_{j}$ to $T_{k}, j=1,2, k=1,2$. Since $\operatorname{ker} \pi_{1}=H_{2}, \operatorname{ker} \pi_{2}=H_{1}$ and $T_{k} \cap H_{j}=\{0\}$, we see that $\pi_{k j}$ is an $N$-isomorphism of $T_{\mathbf{k}}$ into $H_{j}$. However for $k=1,2$,

$$
H_{2}=Y \pi_{2}=\left(T_{k}+H_{1}\right) \pi_{2}=T_{k} \pi_{2}
$$

and similarly

$$
H_{1}=\left(T_{k}+H_{2}\right) \pi_{1}=T_{k} \pi_{1} .
$$

Thus $\pi_{k j}$ is an $N$-isomorphism of $T_{k}, k=1,2$, onto $H_{j}, j=1,2$. Now $\pi_{11}$ is an $N$-isomorphism of $T_{1}$ onto $H_{1}, \pi_{21}^{-1}$ an $N$-isomorphism of $H_{1}$ onto $T_{2}, \pi_{22}$ an $N$-isomorphism of $T_{2}$ onto $H_{2}$ and $\pi_{12}^{-1}$ an $N$-isomorphism of $H_{2}$ onto $T_{1}$. Thus $\pi_{11} \pi_{21}^{-1} \pi_{22} \pi_{12}^{-1}$ (= $\delta$ say) is an $N$-automorphism of $T_{1}$. Since $T_{1}$ is 2-tame it is, by 1.4 of [4], a central sum of $T_{1} \delta$ and $T_{1}(1-\delta)$ (here 1 is the identity on $T_{1}$ ). However, $T_{1} \delta=T_{1}$ and, by 1.3 of $[4], T_{1}(1-\delta)$ is in the centre of $T_{1}$. Thus if $T_{1}(1-\delta) \neq\{0\}$, then $N /\left(0: T_{1}(1-\delta)\right)$ is a non-zero ring. Since $N$ is ring-free, we have $T_{1}(1-\delta)=\{0\}$ and thus

$$
\pi_{11} \pi_{21}^{-1} \pi_{22} \pi_{12}^{-1}=1 \text {. }
$$

Now a typical element $x_{2}$ of $T_{2}$ is of the form $x_{2} \pi_{21}+x_{2} \pi_{22}$. However, since $\pi_{11} \pi_{21}^{-1}$ is an $N$-isomorphism of $T_{1}$ onto $T_{2}$, there exists $x_{1}$ in $T_{1}$ such that $x_{1} \pi_{11} \pi_{21}^{-1}=x_{2}$. Thus 


$$
x_{2}=x_{1} \pi_{11} \pi_{21}^{-1} \pi_{21}+x_{1} \pi_{11} \pi_{21}^{-1} \pi_{22}
$$

which by (c) yields

$$
x_{2}=x_{1} \pi_{11}+x_{1} \pi_{12}=x_{1} .
$$

Thus, $x_{2}$ is in $T_{1}$ and $T_{2} \leqq T_{1}$. Similarly $T_{1} \leqq T_{2}$. Thus $T_{1}=T_{2}$ and the lemma is proved.

To prove the straightforward extension of this lemma, that was mentioned above, an elementary proposition is in order.

Proposition 18. Suppose the $N$-group $Y$ is a direct sum $H_{1} \oplus H_{2}$ of submodules $H_{i}$, $i=1,2$. If $W$ is a submodule of $H_{1}$, then it is a submodule of $Y$.

Material has been covered that provides a key result in establishing Theorem 1.

Lemma 19. Let $N$ be a ring-free near-ring. Suppose the $N$-group $Y$ is a direct sum $H_{1} \oplus H_{2}$ of submodules $H_{j}, j=1,2$. If $T_{k}, k=1,2$, are 2-tame $N$-subgroups of $Y$ such that $T_{1} \cap H_{j}=T_{2} \cap H_{j}$ for $j=1,2$, and $T_{k}+H_{j}=Y$ for $k=1,2, j=1,2$, then $T_{1}=T_{2}$.

Proof. Let $\pi_{1}$ be the projection of $T$ onto $H_{1}$. Since $k e r \pi_{1}=H_{2}$ and $T_{1}+H_{2}=Y$ we have $H_{1}=T_{1} \pi_{1}$ and $H_{1}$ is an $N$-homomorphic image of a 2-tame $N$-group. Thus $H_{1}$ is 2-tame and, by Proposition 18, $T_{1} \cap H_{1}\left(=T_{2} \cap H_{1}\right)$ is a submodule of $Y$. Similarly $\mathrm{T}_{1} \cap \mathrm{H}_{2}\left(=\mathrm{T}_{2} \cap \mathrm{H}_{2}\right)$ is a submodule of $Y$. Let $X$ be the submodule

$$
T_{1} \cap H_{1} \oplus T_{1} \cap H_{2}=T_{2} \cap H_{1} \oplus T_{2} \cap H_{2}
$$

of $Y$. Set $\bar{Y}=Y / X, \bar{H}_{j}=\left(H_{j}+X\right) / X$ for $j=1,2$, and $\bar{T}_{k}=T_{k} / X$ for $k=1,2$. Clearly the $\bar{T}_{k}, k=1,2$, are 2 -tame $N$-subgroups of $\bar{Y}$. If it is shown that $\bar{Y}=\bar{H}_{1} \oplus \bar{H}_{2}, \bar{T}_{k} \cap \bar{H}_{j}=\{0\}$ and $\bar{T}_{k}+\bar{H}_{j}=\bar{Y}$ for $k=1,2$, and $j=1,2$, then it will follow, by Lemma 17 , that $\bar{T}_{1}=\bar{T}_{2}$. This will in turn imply $T_{1}=T_{2}$.

Firstly $\bar{H}_{1}+\bar{H}_{2}=\bar{Y}$, since $H_{1}+H_{2}=Y$. Also the sum $\bar{H}_{1}+\bar{H}_{2}$ is direct, since

$$
\begin{aligned}
\left(H_{1}+X\right) \cap\left(H_{2}+X\right) & =\left(H_{1}+H_{2} \cap T_{1}\right) \cap\left(H_{2}+H_{1} \cap T_{1}\right) \\
& =H_{1} \cap\left(H_{2}+H_{1} \cap T_{1}\right)+H_{2} \cap T_{1} \\
& =H_{1} \cap T_{1}+H_{2} \cap T_{1}=X .
\end{aligned}
$$

Secondly we must show $\bar{T}_{k} \cap \bar{H}_{j}=\{0\}$ for $k=1,2$, and $j=1,2$. Since for $k=1,2$,

$$
T_{k} \cap\left(H_{1}+T_{k} \cap H_{2}\right)=X
$$

it follows that $\bar{T}_{k} \cap \bar{H}_{1}=\{0\}$. Similarly $\bar{T}_{k} \cap \bar{H}_{2}=\{0\}$ and $\bar{T}_{k} \cap \bar{H}_{j}=\{0\}$ for $k=1,2$, and $j=1,2$. Finally it is clearly true that since $T_{k}+H_{j}=Y$ for $k=1,2, j=1,2$, that 
$\bar{T}_{k}+\bar{H}_{j}=\bar{Y}$. From comments above it follows that $T_{1}=T_{2}$. The proof of Lemma 19 is complete.

The proof of Theorem 1 is now accomplished in the concluding analysis that follows.

We shall be assuming that $N$ is a near-ring satisfying the conditions of Theorem 1 . Furthermore, we shall assume that $V_{i}, i=1,2$, are two faithful 2-tame $N$-groups. The fact that the $V_{i}, i=1,2$, are finite follows from Lemma 5 . The remaining requirement is to show that $V_{1}$ is $N$-isomorphic to $V_{2}$. By Lemma $6, V_{i}, i=1,2$, must both have a composition series of length $m(N)$. It will be shown by induction on $m(N)$ that $V_{1}$ is $N$-isomorphic to $V_{2}$. If $m(N)=0$, then, by Lemma $5, V_{i}, i=1,2$, cannot have any minimal $N$-groups. This can only happen if the $V_{i}, i=1,2$, are $\{0\}$ and $N=\{0\}$. In this case $V_{1}$ is $N$-isomorphic to $V_{2}$.

We may therefore assume that $m(N)>0$ and thus, by Lemma 6 , both $V_{i}, i=1,2$, are non-zero $N$-groups. Also $V_{1}$ clearly has a minimal $N$-subgroup. We now use Lemma 9 to exclude that situation where this minimal $N$-subgroup is unique. If such an $N$ subgroup is unique, then, by Lemma $8, N$ has a unique minimal ideal ( $T$ say). By 5 , there exists a minimal right ideal $R \leqq T$ of $N$. However, Lemma 9 implies that both $V_{i}$, $i=1,2$, are $N$-isomorphic to $N / C_{N}(R)$ and $V_{1}$ is therefore $N$-isomorphic to $V_{2}$.

The proof of Theorem 1 has been reduced to showing that when $V_{i}, i=1,2$, are non-zero and $V_{1}$ has more than one minimal $N$-subgroup then $V_{1}$ is $N$-isomorphic to $V_{2}$. In the remainder of the proof a certain amount of extra notation will be required. In supplying this requirement we shall also obtain certain useful equations (see (d) below). Let $U_{j 1}, j=1,2$, be two distinct minimal $N$-subgroups of $V_{1}$. By Lemma $10, U_{11}$ is not $N$-isomorphic to $U_{21}$. Also, by Lemma $11, N$ has a minimal right ideal $N$-isomorphic to $U_{11}$. Again, by Lemma 11, we can find a minimal $N$-subgroup $U_{12}$ of $V_{2}, N$-isomorphic to $U_{11}$. Similarly $V_{2}$ contains a minimal $N$-subgroup $U_{22}, N$-isomorphic to $U_{21}$. Since $U_{12}$ and $U_{22}$ are not $N$-isomorphic, they are clearly distinct. Now take $Y_{i}, i=1,2$, to be the external direct sum of $V_{i} / U_{1 i}$ and $V_{i} / U_{2 i}$. If $H_{1 i}$ is taken to be $\left(V_{i} / U_{1 i},\{0\}\right)$ and $H_{2 i}$ is taken as $\left(\{0\}, V_{i} / U_{2 i}\right)$, then, by 16 , the $H_{j i}, j=1,2, i=1,2$, are submodules of $Y_{i}, i=1,2$, and $Y_{i}=H_{1 i} \oplus H_{2 i}$. Furthermore, by Lemma 16, we can define two $N$-group embeddings $\delta_{i}, i=1,2$, of $V_{i}$ into $Y_{i}$ by setting $v_{i} \delta_{i}=\left(v_{i}+U_{1 i}, v_{i}+U_{2 i}\right)$, for all $v_{i}$ in $V_{i}$. If $X_{1 i}$ is taken as $\left(\left(U_{1 i}+U_{2 i}\right) / U_{1 i},\{0\}\right), \mathrm{i}=1,2$, and $X_{2 i}$ as $\left(\{0\},\left(U_{1 i}+U_{2 i}\right) / U_{2 i}\right)$ then, by Lemma 16, $X_{j i}, j=1,2, i=1,2$, are submodules of $Y_{i}$ and

$$
V_{i} \delta_{i}+H_{j i}=Y_{i} \text { and } \quad V_{i} \delta_{i} \cap H_{j i}=X_{j i}
$$

We now show how the equations (d) can be used in conjunction with certain other equations. If it is shown that there exists an $N$-isomorphism $\tau$ of $Y_{1}$ onto $Y_{2}$ having the properties

$$
H_{j 1} \tau=H_{j 2} \text { and } X_{j 1} \tau=X_{j 2}
$$

then, using (d), Theorem 1 will follow from Lemma 19. The actual construction of $\tau$, which depends on the induction assumption, is postponed until the next paragraph. 
It is sufficient for the moment to show that the existence of such an $N$-isomorphism shows $V_{1}$ is $N$-isomorphic to $V_{2}$. By (d), we have that for $j=1,2, V_{1} \delta_{1}+H_{j 1}=Y_{1}$ and $V_{1} \delta_{1} \cap H_{j 1}=X_{j 1}$ for $j=1,2$. By (e), it follows on applying $\tau$ to these results, we obtain $V_{1} \delta_{1} \tau+H_{j 2}=Y_{2}$, and $V_{1} \delta_{1} \tau \cap H_{j 2}=X_{j 2}$ for $j=1,2$. However, by (d) this implies

$$
V_{1} \delta_{1} \tau+H_{j 2}=V_{2} \delta_{2}+H_{j 2} \quad \text { and } \quad V_{1} \delta_{1} \tau \cap H_{j 2}=V_{2} \delta_{2} \cap H_{j 2} \quad \text { for } j=1,2 \text {. }
$$

Thus if in Lemma 19 we take $Y=Y_{2}, H_{1}=H_{12}, H_{2}=H_{22}, T_{1}=V_{1} \delta_{1} \tau$ and $T_{2}=V_{2} \delta_{2}$, then we obtain $V_{1} \delta_{1} \tau=V_{2} \delta_{2}$. This follows because the $N$-groups $V_{1}$ and $V_{2}$ are 2-tame and, since $\delta_{2}$ and $\delta_{1} \tau$ are $N$-homomorphisms, $V_{1} \delta_{1} \tau$ and $V_{2} \delta_{2}$ are also 2-tame $N$-groups. Thus $V_{1} \delta_{1} \tau=V_{2} \delta_{2}$, where $V_{i}, i=1,2$, is $N$-isomorphic to $V_{i} \delta_{i}$ (the $\delta_{i}$ are embeddings). Since $\tau$ is an $N$-isomorphism, it follows that $V_{1}$ is $N$-isomorphic to $V_{2}$. It only remains to show that there exists an $N$-isomorphism $\tau$ of $Y_{1}$ onto $Y_{2}$ such that (e) holds.

The proof of the existence of $\tau$ will use the induction assumption and previous results. Let $S\left(U_{1 i}\right), i=1,2$, be the right ideals defined as in Lemma 15 . Since $U_{11}$ is $N$-isomorphic to $U_{12}, S\left(U_{11}\right)=S\left(U_{12}\right)$. Thus, by Lemma 15 , we have that $\left(U_{11}: V_{1}\right)=$ $\left(U_{12}: V_{2}\right)$. Thus $V_{i} / U_{1 i}, \quad i=1,2, \quad$ are faithful 2-tame $N /\left(U_{11}: V_{1}\right)$-groups (i.e. $N /\left(U_{12}: V_{2}\right)$-groups). The near-ring $N /\left(U_{11}: V_{1}\right)$ (i.e. $\left.N /\left(U_{12}: V_{2}\right)\right)$ is ring-free. Furthermore, both the $N$-groups $V_{i} / U_{1}, i=1,2$, have a composition series of length $m(N)-1$. By induction, we may assume that $V_{1} / U_{11}$ is $N /\left(U_{11}: V_{1}\right)$-isomorphic (i.e. $N /\left(U_{12}: V_{2}\right)$-isomorphic) to $V_{2} / U_{12}$. Since $\left(U_{11}: V_{1}\right)\left(=\left(U_{12}: V_{2}\right)\right)$ annihilates $V_{i} / U_{1 i}, i=$ 1 ,2, we have that $V_{1} / U_{11}$ is $N$-isomorphic (by $\lambda_{1}$ say) to $V_{2} / U_{12}$. Similarly, $V_{1} / U_{21}$ is $N$-isomorphic (by $\lambda_{2}$ say) to $V_{2} / U_{22}$. We can now find an $N$-isomorphism $\tau$ of $Y_{1}$ into $Y_{2}$ as in the previous paragraph. For any $\left(x_{1}, x_{2}\right)$ in $Y_{1}, x_{1}$ in $V_{1} / U_{11}$ and $x_{2}$ in $V_{1} / U_{21}$, we define the map $\tau$ of $Y_{1}$ into $Y_{2}$ by setting $\left(x_{1}, x_{2}\right) \tau=\left(x_{1} \lambda_{1}, x_{2} \lambda_{2}\right)$. Clearly $\tau$ is an $N$-isomorphism of $Y_{1}$ onto $Y_{2}$. Since $H_{11}$ is simply the submodule (see Lemma 16) $\left(V_{1} / U_{11},\{0\}\right)$ of $Y_{1}$, it follows that $H_{11} \tau=H_{12}$. Similarly $H_{21} \tau=H_{22}$. Thus the first set of equations in (e) hold. It remains to show that $X_{j 1} \tau=X_{j 2}$ for $j=1,2$. Now $X_{11}=\left(\left(U_{11}+U_{21}\right) / U_{11},\{0\}\right)$ and this submodule of $Y_{1}$ is clearly $N$-isomorphic to $U_{21}$. Thus $X_{11} \tau$ is a submodule of $Y_{2}, N$-isomorphic to $U_{21}$. However $X_{11} \tau \leqq H_{11} \tau=H_{12}$. Thus $X_{11} \tau$ is a submodule of $H_{12}, N$-isomorphic to $U_{21}$ (i.e. to $U_{22}$ ). Since $H_{12}$ is $N$-isomorphic to $V_{2} / U_{12}, H_{12}$ is a 2-tame $N$-group. Since $X_{12}=\left(\left(U_{12}+U_{22}\right) / U_{12},\{0\}\right)$ is $N$-isomorphic to $U_{22}$ it follows, by Lemma 10 , that $X_{11} \tau=X_{12}$. Similarly $X_{21} \tau=X_{22}$. The second set of equations in (e) also hold. From the previous paragraph $V_{1}$ is $N$-isomorphic to $V_{2}$ and Theorem 1 is completely proved.

Acknowledgement. The author takes this opportunity to express his gratitude for the use of facilities in the Department of Mathematics at Auckland, enabling him to finish this paper. I am very much indebted to Marilyn Talamaivao for her effort to reproducing this material.

\section{REFERENCES}

1. C. W. CURTIS and I. ReINER, Representation Theory of Finite Groups and Associative Algebras (Interscience Publishers, New York 1966). 
2. J. D. P. Meldrum, Near-rings and their Links with Groups (Pitman Publishing, London (1985).

3. G. PiLz, Near-rings (North-Holland, Amsterdam 1983).

4. S. D. Scort, Linear $\Omega$-groups, polynomial maps, Contr. Gen. Alg. 8 (1991), 239-293.

5. S. D. Scotr, Tame near-rings and N-groups, Proc. Edinburgh Math. Soc. 23 (1980), 275-296.

6. S. D. Scotr, Tame Theory, unpublished booklet.

Department of Mathematics

UNIVERSITY OF AUCKLAND

Private Bag 92019

AUCKLAND

New Zealand 